\title{
Electronic properties of deep levels in $p$-type CdTe
}

\author{
R. T. Collins and T. C. McGill \\ California Institute of Technology, Pasadena, California 91125
}

(Received 1 March 1983; accepted 25 May 1983)

\begin{abstract}
DLTS and associated electrical measurements were made on unintentionally doped CdTe crystals obtained from several vendors, on $\mathrm{Cu}$-doped CdTe, and on Te-annealed CdTe. All of the crystals were $p$-type. Four majority carrier deep levels were observed in the temperature range from $100-300 \mathrm{~K}$ with activation energies relative to the valence band of $0.2,0.41,0.45$, and 0.65 $\mathrm{eV}$. Two of these levels were specific to certain crystals while the other two were seen in every sample and are attributed to common impurities or native defects. Fluctuations in the concentrations of levels across samples and as a result of modest sample heating $(400 \mathrm{~K})$ were also observed.
\end{abstract}

PACS numbers: 72.80.Ey, 72.20.Jv, 71.55.Fr

\section{INTRODUCTION}

The electronic properties of II-VI compound semiconductors are not well understood. The situation is complicated by the presence of native defects and defect complexes, in addition to impurities incorporated into the crystals during growth. CdTe is a II-VI semiconductor which is of recent interest because it serves as a substrate for the growth of the lattice-matched ternary $\mathrm{Hg}_{1-x} \mathrm{Cd}_{x} \mathrm{Te}$. It has also been proposed for.use as a nuclear detector and in solar cells. In all of these applications an understanding of the deep-level properties of the material is important to successfully fabricating devices from CdTe. There have been a number of studies of deep levels in CdTe. ${ }^{1}$ The results of early measurements are hard to compare because of the variety of techniques and samples used. Recently, more sensitive and reproducible deep-level transient spectroscopy (DLTS) measurements have been made on $n$-type CdTe, but the CdTe typically used for the above applications is unintentionally doped, asgrown material which is generally $p$-type ${ }^{2-4}$ Very little information about deep levels in these crystals exists.

This paper presents the results of a study of the majority carrier deep levels in $p$-type CdTe using the technique of DLTS. The experimental method will be described, and the properties of each sample studied will be discussed. Results of the measurements will be presented providing a comparison of as-grown CdTe crystals obtained from various sources. Information on the deep levels in CdTe doped with $\mathrm{Cu}$ and $\mathrm{CdTe}$ which had been annealed in Te vapor will also be given. Finally, possible explanations for the observations that were made and conclusions based on these observations will be suggested.

\section{EXPERIMENTAL}

DLTS and $\mathrm{C}-\mathrm{V}$ measurements were made on six different crystals of $p$-type CdTe. All of the crystals were examined in an as-grown condition. One was also annealed in Te vapor prior to the measurements. The history and properties of each of these materials is given in Table I. Shallow level concentrations $\left(N_{A}-N_{D}\right)$ taken from reverse bias capacitance measurements are also given in Table I. Five of the six crystals were unintentionally doped. It is not known if the observed shallow level concentrations in these crystals, which are quite high, were due to residual impurities or to stoichiometry. The latter is suspected. Crystal E was made from material which was taken from crystal $B$ and annealed at $800{ }^{\circ} \mathrm{C}$ for $\approx 2 \mathrm{~h}$ in a sealed, evacuated quartz ampule containing elemental $\mathrm{Te}$. Crystal $\mathrm{F}$ was $\mathrm{CdTe}$ doped with $\mathrm{Cu}$ at a level of $10^{16} \mathrm{~cm}^{-3}$.

The measurements described here were performed on Schottky barrier devices fabricated on samples taken from the crystals. Both $\mathrm{Au}$ and $\mathrm{Cd}$ barriers were used. $\mathrm{Au}$ and $\mathrm{Cd}$ Schottky barriers were prepared in two different ways. In the first method, $160 \mu \mathrm{m}$ diameter dots were evaporated onto air-cleaved $\{110\}$ surfaces in an ion pumped vacuum system at $10^{-6}$ Torr. $\mathrm{Ni}$ or a $\mathrm{Cu} / \mathrm{Au}$ alloy was evaporated onto the rear face of the sample following an etch in $\mathrm{K}_{2} \mathrm{CrO}_{7}$ and $\mathrm{H}_{2} \mathrm{SO}_{4}$ solution to provide the ohmic contact. ${ }^{5}$

In the second method of preparation, $\mathrm{Au}$ or $\mathrm{Cd}$ was evaporated onto polished $\{111\}$ sample surfaces which had been etched for $5 \mathrm{~min}$ in a $0.5 \% \mathrm{Br}$-Methanol solution. These dots were $500 \mu \mathrm{m}$ in diameter. Again, evaporated Ni or $\mathrm{Cu} / \mathrm{Au}$ alloy provided the $\mathrm{Ohmic}$ contact.

Reverse bias capacitance characteristics for samples prepared as described above were recorded at room temperature using a model 71A Boonton capacitance meter with a $15 \mathrm{mV}, 1 \mathrm{MHz}$ test signal. DLTS spectra were taken on the

TABLE I. Shallow level concentrations and histories of each of the CdTe crystals used in the DLTS measurements.

\begin{tabular}{|c|c|c|}
\hline Crystal & $\begin{array}{l}\text { Shallow level } \\
\text { concentration }\end{array}$ & Comments \\
\hline $\mathbf{A}$ & $5-8 \times 10^{15} \mathrm{~cm}^{-3}$ & Bridgman growth by II-VI Corp. \\
\hline $\mathbf{B}$ & $6 \times 10^{14} \mathrm{~cm}^{-3}$ & $\begin{array}{l}\text { Traveling heater method by Radiation } \\
\text { Monitor. }\end{array}$ \\
\hline $\mathrm{C}$ & $2-3 \times 10^{16} \mathrm{~cm}^{-3}$ & Bridgman growth by Rockwell Int. \\
\hline D & $1.5 \times 10^{15} \mathrm{~cm}^{-3}$ & Bridgman growth by II-VI Corp. \\
\hline $\mathbf{E}$ & $>2 \times 10^{16} \mathrm{~cm}^{-3}$ & $\begin{array}{l}\text { Crystal " } \mathrm{B} \text { " annealed in Te vapor at } \\
800^{\circ} \mathrm{C} \approx 2 \mathrm{~h} \text {. }\end{array}$ \\
\hline $\mathrm{F}$ & $1.2 \times 10^{16} \mathrm{~cm}^{-3}$ & $\begin{array}{l}\text { Bridgman growth by II-VI Corp. Doped } \\
\text { with } 10^{16} \mathrm{~cm}^{-3} \mathrm{Cu} \text {. }\end{array}$ \\
\hline G & $6 \times 10^{15} \mathrm{~cm}^{-3}$ & Bridgman growth by Texas Instruments. \\
\hline
\end{tabular}


same samples using a fast capacitance bridge and double boxcar integrator as described by Lang. ${ }^{6}$

Photoresponse and I-V measurements were also made on the Au and Cd Schottky barriers prepared on the air-cleaved $\{110\}$ sample surfaces to determine the Schottky barrier heights.

\section{RESULTS}

Results of the DLTS measurements on the various crystals are presented below. Minority carrier injection is negligible in Schottky barrier devices if the barrier height is considerably less than the band gap, as is the case for $\mathrm{Cd}$ and $\mathrm{Au}$ on $p$-type CdTe. ${ }^{7}$ For this reason, only majority carrier traps were observable in this study. No differences in the spectra were evident as a result of the method of device preparation or the Schottky barrier used.

As shown in Figs. 1-4, four major deep levels were seen in the crystals in the temperature range from $100-300 \mathrm{~K}$. Deep level $\mathrm{H} 1$ was seen in $\mathrm{A}$ (Fig. 1) and D (Fig. 2) at concentrations of $1 \times 10^{14}$ and $3 \times 10^{13} \mathrm{~cm}^{-3}$, respectively. This level may have been present in $\mathrm{F}$ but at a very low concentration. The low temperature region where $\mathrm{H} 1$ occurred was scanned for all the crystals, although it is only shown for $\mathbf{A}$ and $\mathbf{D}$. Level $\mathrm{H} 2$ was only seen in crystal C (Fig. 2). Its concentration was between $2 \times 10^{13}$ and $3 \times 10^{14} \mathrm{~cm}^{-3}$. Levels $\mathrm{H} 3$ and $\mathrm{H} 4$ were seen in all of the crystals (Figs. 1-4). Level H3 appeared over approximately a $5 \mathrm{~K}$ range of temperatures in the samples studied. This variation was even observed in samples taken from the same crystal. This suggests that $\mathrm{H3}$ may really be due to more than one level, and relative concentration changes in the levels caused the position of $\mathrm{H} 3$ to shift. The concentrations of $\mathrm{H} 3$ in crystals $A$ through $\mathrm{D}$ were $4 \times 10^{13}-4 \times 10^{14}, 1 \times 10^{12}, 3 \times 10^{13}-3 \times 10^{14}$ and $2 \times 10^{13}$ $1 \times 10^{14} \mathrm{~cm}^{-3}$, respectively. In $\mathrm{G}$ (Fig. 4 ) its concentration

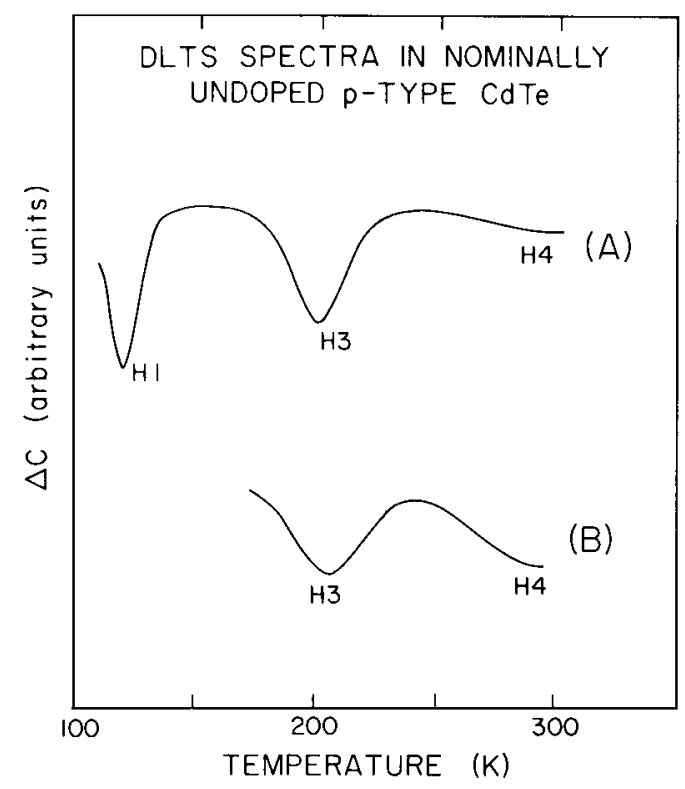

FIG. 1. Characteristic DLTS spectra for hole traps in nominally undoped, $p$ type CdTe crystals $A$ and $B$. The positions of the boxcar windows during the scans were $t_{1}=5 \mathrm{~ms}$ and $t_{2}=45 \mathrm{~ms}$.

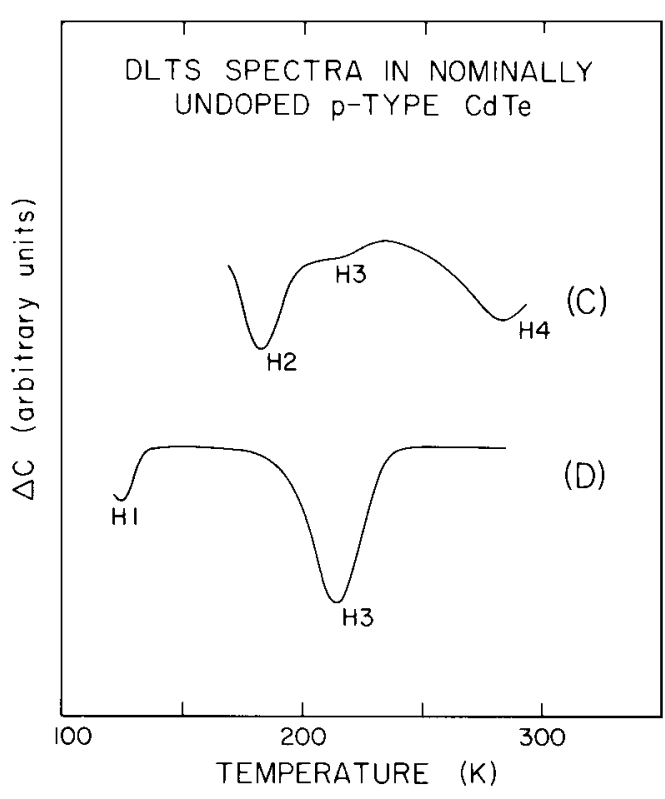

FIG. 2. Characteristic DLTS spectra for hole traps in nominally undoped, $p$ type CdTe crystals $\mathrm{C}$ and $\mathrm{D}$. The positions of the boxcar windows during the scans were $t_{1}=5 \mathrm{~ms}$ and $t_{2}=45 \mathrm{~ms}$.

was $4 \times 10^{12} \mathrm{~cm}^{-3} \cdot \mathrm{H} 3$ in $\mathrm{E}$ and $\mathrm{F}$ will be discussed below. There was a lot of fluctuation in the trap concentrations, as can be seen from the above values. These fluctuations even occurred from device to device on the same sample. In the case of level $\mathrm{H} 4$ this effect was more pronounced. At the sensitivity used in the spectrum chosen for D in Fig. 2, the level was not visible, although, in other cases, its concentration matched that of H3. These deep trap concentrations were estimated from the formula $2 \Delta C / C=N_{d} / N_{s}$ where $C$ is the capacitance of the diode, $\Delta C$ is the change in capacitance caused by completely filling the deep level, $N_{d}$ is the deep level concentration, and $N_{s}$ is the shallow level concen-

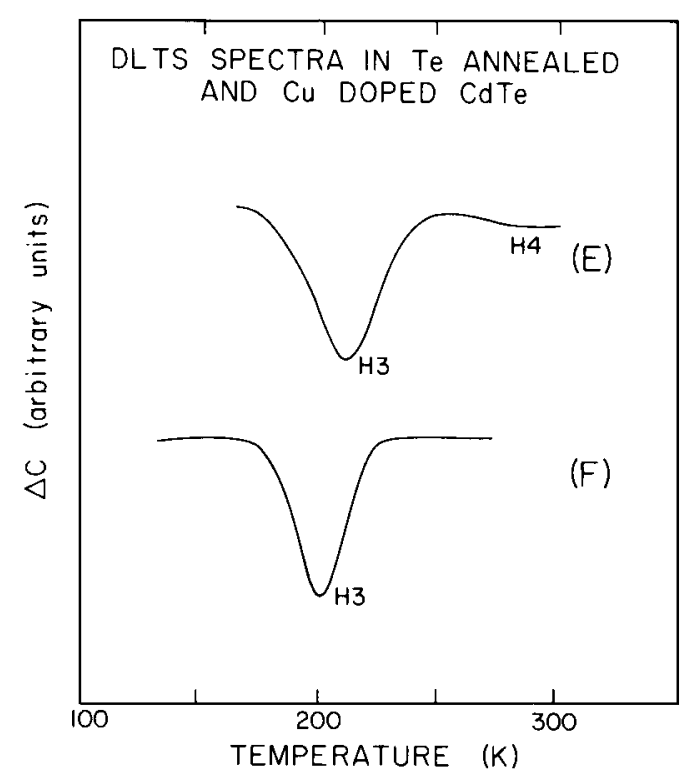

FIG. 3. Characteristic DLTS spectra for Te-annealed (E) and Cu-doped (F) p-type CdTe. The positions of the boxcar windows during the scans were $t_{1}=5 \mathrm{~ms}$ and $t_{2}=45 \mathrm{~ms}$. 


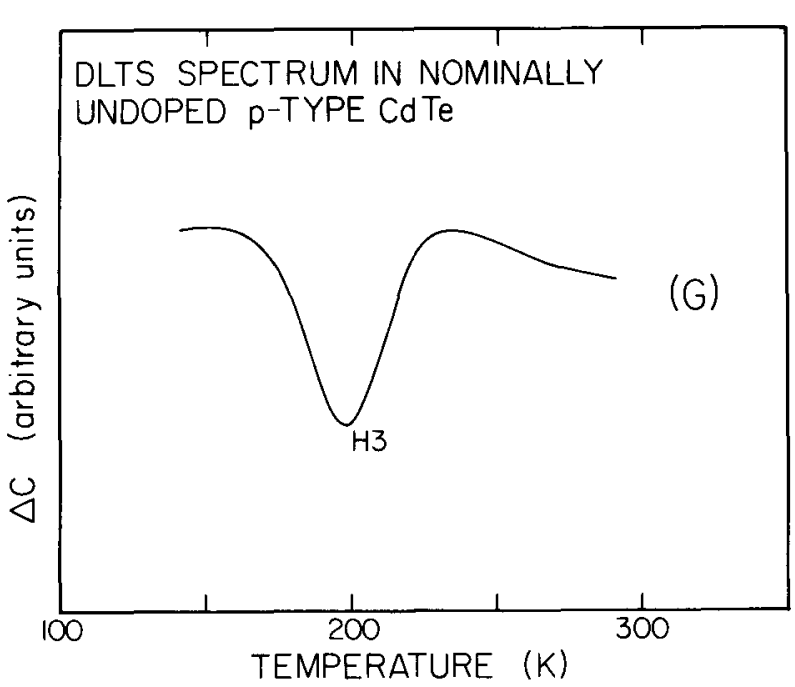

FIG. 4. DLTS spectra for hole traps in nominally undoped, p-type CdTe crystal G. The positions of the boxcar windows during the scans were $t_{1}=5$ $\mathrm{ms}$ and $t_{2}=45 \mathrm{~ms}$.

tration. ${ }^{6}$ Edge region effects are neglected using this method, but, such accuracy is not necessary in light of the large variations in trap concentrations which were mentioned above.

Material E was CdTe which had been taken from crystal B and annealed in $\mathrm{Te}$ vapor at $800^{\circ} \mathrm{C}$ for about $2 \mathrm{~h}$. The anneal caused the shallow level concentration to increase by two orders of magnitude. As can be seen from Fig. 3, H3 and H4 were still present. H3's concentration was approximately $5 \times 10^{14} \mathrm{~cm}^{-3}$. This value is also about two orders of magnitude higher than prior to the anneal.

Crystal $\mathrm{F}$ had been doped with $\mathrm{Cu}$ at about $10^{16} \mathrm{~cm}^{-3}$. This concentration is close to the shallow level concentration obtained from the $\mathrm{C}-\mathrm{V}$ profile and may indicate that the $\mathrm{Cu}$ dopants were not strongly compensated by native defects. The DLTS spectrum for F, as seen in Fig. 3, contains $\mathrm{H} 3$ at a concentration of $4 \times 10^{13} \mathrm{~cm}^{-3}$, a value similar to that found in the undoped crystals. $\mathrm{H} 4$ was also present at a much lower concentration and is not seen in the spectrum shown. Attempts were also made to dope crystals with $\mathrm{Cu}$ following procedures outlined by Ref. 8. Again, there were no new levels or significant enhancements of existing levels.

Other trapping states were visible in the above room temperature range of the spectra, but, after the samples were heated to $400 \mathrm{~K}$ in the process of taking the data, changes occurred in the DLTS spectra. Levels which were seen above room temperature left completely. Some of the below room temperature levels also suffered concentration increases or decreases of as much as an order of magnitude. The direction of the change was not always the same. For this reason no above room temperature spectra are given. The DLTS spectra shown are for unheated samples immediately after preparation.

Arrhenius plots for levels $\mathrm{H} 1-\mathrm{H} 4$, along with their associated activation energies, are given in Fig. 5. Capture cross sections for the various states were not directly measurable with our system because, for the shortest reduced bias pulse we could apply ( $\approx 20 \mathrm{~ns})$, all the levels were completely filled with holes. From this we can place a lower bound of
$5 \times 10^{-14} \mathrm{~cm}^{2}$ on the capture cross section for holes, a value which is in reasonable agreement with that determined from the $y$ intercepts of the Arrhenius plots. Because of this, the activation energies in Fig. 5 have not been corrected for temperature dependent cross sections. No correction for electric field effects has been applied either. The data used for $\mathrm{H} 3$ in Fig. 5 is for a particular device. If other devices are used, the temperature fluctuations mentioned above cause the line to shift, but the slope stays within the error range given.

$\mathrm{Au}$ and $\mathrm{Cd}$ barrier heights on the $\{110\}$ air-cleaved surface of the CdTe were found to be approximately 0.6 and 1.0 $\mathrm{eV}$, respectively. The Au value is in agreement with previous measurements. ${ }^{9}$ The value for the Cd barrier height is approximately equal to the CdTe band gap minus the $\mathrm{Cd}$ barrier height on $n$-type CdTe taken from Ref. 10.

\section{CONCLUSIONS}

Impurities and native defects are both potential sources of deep levels in CdTe. ${ }^{1}$ Although the crystal defects responsible for the deep levels observed in this study cannot be directly identified, it is possible to draw some conclusions about them based on the results of these measurements. Levels $\mathrm{H} 1$ and $\mathrm{H} 2$ were specific to certain crystals and are, therefore, likely candidates for impurities. Levels $\mathrm{H} 3$ and $\mathrm{H} 4$, on the other hand, were present in all of the crystals. They could be the result of a common impurity, native defects, or even grosser crystal defects (such as Te precipitates). There have been previous reports of levels approximately $0.3 \mathrm{eV}$ from the valence band that were due to $\mathrm{Cu}, \mathrm{Au}$ and $\mathrm{Ag} .{ }^{11,12}$ Identifications were based on associating the levels that were observed with the crystal dopants. Although $\mathrm{Au}$ and $\mathrm{Ag}$ diffusions have yet to be tried, the present work seems to indicate that the levels we observe are not due to $\mathrm{Cu}$, since the presence of $\mathrm{Cu}$ in crystal $\mathrm{F}$ did not enhance any of the levels seen and because the attempts at $\mathrm{Cu}$ doping did not significantly

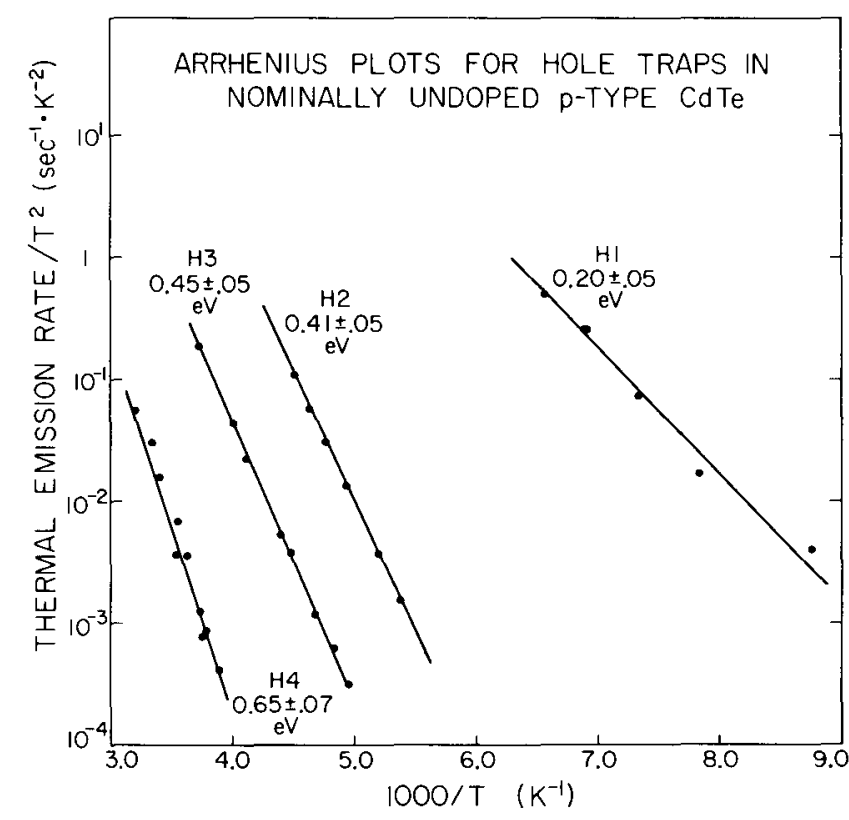

Fig. 5. Arrhenius plots for the four majority carrier deep levels seen in DLTS measurements on $p$-type CdTe. 
increase the concentrations of any of the traps. It is possible that there are deep levels associated with the $\mathrm{Cu}$, but that the temperature range of this study did not include $\mathrm{Cu}$-related peaks. This remains to be tested. The fact that $\mathrm{H} 3$ and $\mathrm{H} 4$ along with $\left(N_{A}-N_{D}\right)$ were effected by the Te anneals suggests that native defects are responsible.

Concentrations of deep levels in as-grown crystals were generally $10^{14} \mathrm{~cm}^{-3}$ or less. Although this is quite low, fluctuations were also seen in the concentrations of traps from device to device on a given sample. Even more disturbing were the changes in peak amplitudes produced by very modest sample heating $(400 \mathrm{~K})$. No attempts were made to determine if similar concentration changes occurred for samples heated before device fabrication, but since the same levels were observed for both $\mathrm{Au}$ and $\mathrm{Cd}$ barriers, it is unlikely that the changes occurred as a result of diffusion of barrier metal into the active region of the CdTe diode.

In summary, we have made DLTS measurements on $p$ type CdTe crystals. Four deep levels were observed (H1-H4) in the temperature range from $100-300 \mathrm{~K}$. Levels $\mathrm{H} 1$ and $\mathrm{H} 2$ only occurred in a few of the samples leading us to believe they are associated with impurities in the crystals. H3 and H4 are in every crystal and may be related to native defects. Measurements on $\mathrm{Cu}$-doped samples indicate that the levels seen are not due to residual $\mathrm{Cu}$ in the $\mathrm{CdTe}$. We also noted variations in the concentrations of the observed levels from device to device on a given sample and significant changes in trap concentrations as a result of modest sample heating.

\section{ACKNOWLEDGMENTS}

The authors wish to acknowledge Rockwell International and Texas Instruments for providing the CdTe used in this research. This work was supported in part by the Army Research Office under Contract No. DAAG29-80-C-0103.

'K. Zanio, in Semiconductors and Semimetals, edited by R. K. Willardson and A. C. Beer (Academic, New York, 1978), Vol. 13.

${ }^{2}$ T. Takebe, J. Saraie, and H. Matsunami, J. Appl. Phys. 43, 5 (1982).

${ }^{3}$ R. T. Collins, T. F. Kuech, and T. C. McGill, J. Vac. Sci. Technol. 21, 191 (1982).

${ }^{4}$ D. Verity, D. Shaw, F. J. Bryant, and C. G. Scott, J. Phys. C 15, 573 (1982).

${ }^{5}$ T. Anthony, A. L. Fahrenbruch, and R. H. Bube, J. Electron. Mater. 2, 89 (1982).

${ }^{6}$ D. V. Lang, J. Appl. Phys. 45, 3023 (1974).

${ }^{7}$ S. M. Sze, Physics of Semiconductor Devices (Wiley, New York, 1981), p. 265.

${ }^{8}$ J. P. Chamonal, E. Molva, and J. L. Pautrat, Solid State Commun. 43, 801 (1982).

${ }^{9}$ J. P. Ponpon, M. Saraphy, E. Buttung, and P. Siffert, Phys. Status Solidi A 59, $259(1980)$.

${ }^{10}$ T. F. Kuech, J. Appl. Phys. 52, 4874 (1981).

"D. de Nobel, Philips Res. Rep. 14, 361 (1959).

${ }^{12}$ M. R. Lorenz and B. Segall, Phys. Lett. 7, 18 (1963). 